\section{Quantum chemistry}

Quantum Chemistry.(Reference Edition.) By J. P. Lowe. Pp. 599. (Academic: New York, San Francisco and London, 1978.) $\$ 49.50 ; £ 32.15$.

QUANTUM CHEMISTRY signifies different things to different minds. Some forget the 'chemistry' and provide for chemists simplified, or simply curtailed, versions of books on quantum mechanics. Some concentrate on molecular properties with and without spectroscopy; others concentrate on molecular structure. The last are increasingly based on computer techniques-as they should be-for they have provided the most important quantitative advances in recent years. In them lies the hope of simulating more precisely the processes that occur when molecules undergo the dramatic changes that result in reaction, and in them lies one of the very few tests we possess of the qualitative ideas that underlie our understanding of the equilibrium geometries of molecules as well as their reaction characteristics.

Dr Lowe has written a book that covers parts of this well trodden ground. It is aimed principally at readers whose interest is in molecular structure, and to say what is not in it may be as helpful as saying what is in it: thus it is not a spectroscopy book, nor is it a molecular properties book. It does, however, deal with basic quantum mechanics (but reserves operator properties until well on into the text), atomic structure, and then, for over half the book, molecular structure. The level of treatment of molecules is slightly beyond what undergraduates normally meet, and covers basic LCAO-MO theory, Hückel theory, and SCF-MO theory. The final chapter, which I suspect the author is proud of most, is on "qualitative molecular orbital theory', which turns out to be the Walsh-type argument for structure and Woodward-Hoffmann rules for reactivity.

Although the basic material is treated in other books with similar titles, there are aspects of the treatment that make the book attractive. The author clearly has an eye for the interesting analogy and felicitous example, and readers will enjoy the sensitivity of his exposition. There are places where he apparently has not troubled to think up an explanation (for example, the Hund rules), but on the whole readers will find themselves in the presence of an imaginative and reliable interpreter.

At various points I thought the author's judgement faltered. For instance, I think very little purpose is served by working through in detail the old-fashioned way of solving the harmonic oscillator Schrödinger equation; and even less is served by sketching the hydrogen atom solution before talking about angular momentum, leaving angular momentum itself largely unexplained, and being unhelpful about spin. Hückel theory is old hat, but it was nice to see modern judgements (and comparison with experiments, as in hyperfine coupling constants) brought into the discussion. Various nice examples of perturbation theory are presented, and group theory is

\section{Insect behaviour}

Insect Behavior. By R. W. Matthews and J. R. Matthews. Pp. 507. (John Wiley: Chichester, UK, and New York, 1978.) £15.85.

IT is rare to find a major gap in the present plethora of scientific books but a treatise on insect behaviour has been badly needed for years. There is surely more published work on this subject than on the behaviour of any other animal group. A great part is descriptive and no small part anecdotal but insect behaviour has also been the subject of innumerable analytical studies, the special strength of which has been their leaning toward physiology rather than psychology.

This book makes a bold and within its purpose successful attempt to fill the gap. Faced with the superabundance of information and the infinite diversity of insect behaviour the authors had to choose between breadth and depth, and chose breadth. Comprehensiveness probably was in fact the most pressing requirement for a first book in the field, one that would serve as a detailed 'guidebook' for entomologists and behaviourists generally.

Inevitably it will be said that in trying to cover everything the authors succeed in saying little about anything. Again and again the reader is tantalised by the opening of an interesting topic only to find it closed again for another. However, while holding to their comprehensive purpose the authors have gone some way to meeting this criticism by frequently interpolating fuller 'case-studies', and by ending every small subsection with citation of up-to-date reviews and books on that specific theme, an invaluable feature. It is difficult to think of any topic that does not get some introductory treatment in the book: slave-making and Zeitgebers, cannibalism and coyness, heritability, flicker-fusion, the $r-K$ spectrum, Solanum alkaloids, stimulus filtering, bioluminescence, parental investment it is all there. The large Subject Index unfortunately does not adequately underpin the guidebook func- presented in a suitably pragmatic way.

In summary, Dr Lowe has written a nice book, which covers old ground in a pedagogically attractive and largely contemporary way. Readers will find it an agreeable, accessible commentary on modern structural quantum chemistry.

P. W. Atkins

P. W. Atkins is Lecturer in Physical Chemistry at the University of $O x$ ford, $U K$.

tion, the bulk of it being insect names. Let us hope the authors will provide a fuller one in future editions.

The authors describe the general approach of their book as "ethological", meaning a primary concern with descriptions, functions, behavioural ecology and evolution. After an introductory chapter including a warning against anthromorphism and teleology, one chapter of 40 pages deals sketchily with nervous and hormonal mechanisms. The rest of the book is devoted to the gamut of behavioural activities under two main heads, "maintenance activities" of individuals, and "communicatory activities" between individuals of the same or different species. Maintenance is covered in chapters on "Spatial Adjustment" (locomotion, orientation, dispersal and migration) and "Feeding Behavior" (plant relations, food location, social feeding, food recognition and feeding regulation).

Communicatory activities take up the remaining 300 pages out of the total of 500 , an emphasis reflecting the enormously increased recent academic and practical interest in these matters. Chemical. visual and mechanical (including acoustic) communication each have a chapter followed by three more strictly focussed on activity types, defensive and reproductive behaviour. brood care and social life. The arrangement is novel and effective. Illustrations are profuse, one on almost every other page, and include many photographs which have not all reproduced well. The reviewer has found one or two erroneous passages and it would be astonishing if there were not more in such an ambitious undertaking. There is naturally a certain transatlantic bias: it is strange, for example, not to find F. Huber's work in an account of acoustic communication.

The book remains a monumental guidebook which insect and other behaviourists will want to have access to for years to come.

\section{J. S. Kennedy}

I. S. Kennedy is Senior Research Fellow and Emeritus Professor of Animal Behaviour at Imperial College of Science and Technology, University of London, $U K$. 